\title{
In Vitro Release Testing of PLGA Microspheres with Franz Diffusion Cells
}

\author{
Laura C. Herrera, María V. Defain Tesoriero, and Laura G. Hermida* \\ Centre of Research and Development in Chemistry, National Institute of Industrial Technology, \\ Avenida General Paz 5445 (B1650WAB), San Martín, Buenos Aires, Argentina
}

e-mail:Ihermida@inti.gob.ar

\begin{abstract}
In the present study, two methods were used to evaluate the in vitro release of leuprolide acetate (LA) from poly(lactide-co-glycolide) (PLGA) microspheres: Franz diffusion cells, typically referred to as "vertical diffusion cells" (VDC), and rotating bottle apparatus (RBA), both modified with a dialysis membrane. This hydrosoluble peptide was chosen as a model drug to study different possibilities of in vitro testing and analyze the variables that affect drug release, respecting sink and physiological conditions. Microspheres were prepared with a conventional double emulsion-solvent evaporation method using PLGA (50:50) with a relatively low molecular weight. Comprehensive stability tests for LA were performed in the conditions used for in vitro release assays. In phosphate-buffered saline (PBS), LA showed no significant degradation, but in an acidic medium, it degraded dramatically. The release profile of the delivery system was governed mainly by diffusion as explained by the low molecular weight of the polymer and the high water solubility of the peptide. The in vitro release profiles were triphasic in vertical diffusion cells and biphasic in the rotating bottle apparatus. The release kinetics was enhanced in RBA with respect to VDC, probably because the constant movement of a suspension of loose microspheres in a large volume and the large membrane area facilitated drug migration. The smoother, triphasic profiles obtained with VDC can be explained by the partial confinement of microspheres, which is similar to the described in vivo behavior of an injectable delivery system.
\end{abstract}

\section{INTRODUCTION}

$$
\text { T: }
$$

he advent of modified-release delivery systems brought the complex issue of in vitro release evaluation, which has not yet been fully solved. Many devices and methods have been tested to clarify the matter and set specifications. Because not all dosage forms should fulfill the same requirements, this becomes even more difficult. For microspheres, much has been done, but there is still disagreement about the best in vitro release testing method to apply. Drugs and polymers of different natures, microsphere features, in vitro release devices, receptor media, and sink conditions are some of the issues that may be encountered during decisionmaking. Many authors have analyzed and discussed the suitability of different devices to perform the release tests of prolonged-release systems $(1,2)$. For instance, USP Apparatus 4, which is based on flow-through cells, was successfully used to obtain release profiles of dexamethasone from long-term, modified-release formulations (3). This is in accordance with USP recommendations about the suitability of this equipment for delivery systems containing drugs with limited solubility (4). Dialysis tests performed with different devices may be useful for testing biodegradable microspheres (5). Different types of shaking and rotating devices have also been widely used for this purpose (6). Franz diffusion cells, typically referred to as "vertical diffusion cells" (VDC), were initially intended for skin permeation. They were further modified to evaluate nasal inserts and other mucosal dispersed systems (7-9).

*Corresponding author.
Another attempt to develop in vitro tests for microspheres was the elevated temperature accelerated assay, which is useful mainly for batch-to-batch comparisons of longacting dosage forms, but they reflect neither the real-time release rate nor the involved mechanism $(3,10,11)$.

After the patent for the leuprolide-polylactic-co-glycolic acid (LA-PLGA) delivery system was issued in the late 1980 s, it was studied extensively $(12,13)$. The release profile of the drug may be influenced by many parameters such as physicochemical properties and drug loading, variations of polymer molecular weight, lactic-to-glycolic ratio, microencapsulation conditions, and in vitro test protocols $(14,15)$. This particular system usually shows a triphasic release profile characterized by an initial burst of the drug near the surface or associated with pores after polymer wetting, usually defined as the amount released during the first $24 \mathrm{~h}$ (15), a lag phase until sufficient polymer erosion has taken place, and a secondary burst with approximately zero-order release kinetics (16-18). This feature generally applies for all cases, but polymer molecular weight, glass-transition temperature $\left(T_{\mathrm{g}}\right)$, drug properties, and even device geometry play important roles in precisely defining the release mechanism of a given delivery system. When a low molecular weight PLGA polymer is employed, for instance, the release is ruled mostly by diffusion. Zolnik and Burgess (19) explained that PLGA degrades from inside to outside at physiological $\mathrm{pH}$. Degradation begins with water going inward; hydrolysis leads to the production of acidic oligomers, which are retained within the microspheres because of the relative hydrophobicity of the polymer, and the phenomenon finally influences the degradation mechanism. When PLGA 
is surrounded by a low $\mathrm{pH}$ medium, it becomes prone to autocatalysis, which is also determined by the size and porosity of PLGA microspheres. Moreover, tissue reaction at the site where parenteral biopolymer-based delivery systems are injected has to be considered (20). In vitro assays should be as predictive as possible of physiological events to achieve an accurate IVIVC. Still, controversy even surrounds the selection of media that resemble in vivo conditions most precisely. Although it has been highly recommended to rely on USP apparatus already proven for robustness, such as the USP Apparatus 4 equipment already mentioned $(21,22)$, the quest for new suitable easy-to-perform in vitro techniques still continues.

The aim of this work is to propose a reliable in vitro procedure that mimics in vivo conditions for the assessment of LA release from PLGA microspheres. Two methods based on vertical diffusion cells and rotating bottle apparatus have been tested to determine the in vitro release of a water-soluble model drug from polymeric microspheres. Both systems were modified with dialysis membranes that separated the microspheres from the external release medium, allowing the migration of the drug without restriction. As leuprolide solubility in water is very high, no violation of sink conditions was suspected in any of the proposed methods. Both methods were studied in different conditions and accompanied by stability tests of leuprolide in the chosen media.

\section{MATERIALS AND METHODS \\ Materials}

Leuprolide acetate was obtained from Bachem (Bubendorf, Switzerland). Poly(D,L-lactide-co-glycolide) (PLGA) was Resomer RG502H (50\% D,L-lactide and 50\% glycolide) with an inherent viscosity of $0.16-0.24 \mathrm{dL} / \mathrm{g}$ and 7,000-17,000 MW range (Boehringer Ingelheim, Ingelheim am Rhein, Germany). Gelatin, polyvinyl alcohol (PVA), methylene chloride, dimethylsulfoxide, Tween 80 , phosphate and sodium salts, and all other reagents were of analytical quality.

\section{Preparation and Characterization of Microspheres}

PLGA microspheres containing LA were prepared by a w/o/w emulsion. PLGA $(8.0 \mathrm{~g})$ was dissolved in $11 \mathrm{~mL}$ of methylene chloride. Leuprolide acetate $(0.907 \mathrm{~g})$ was added to a mixture of gelatin $(0.16 \mathrm{~g})$ and water $(1 \mathrm{~mL})$, previously prepared. A w/o emulsion was formed by mixing the aqueous leuprolide solution into the organic phase using a homogenizer DIAX 900 (Heidolph, Schwabach, Germany) at 22,000 rpm for $10 \mathrm{~min}$ in 30-sec intervals in an ice water bath. The w/o emulsion was added to a $1 \%$ PVA solution $(400 \mathrm{~mL})$ and homogenized at $22,000 \mathrm{rpm}$ for $10 \mathrm{~min}$. The obtained w/o/w emulsion was stirred at $2,000 \mathrm{rpm}$ for $1 \mathrm{~h}$ at $25^{\circ} \mathrm{C}$ to allow the methylene chloride to evaporate. After evaporation of the organic solvent, the resulting microspheres were filtered through a $100-\mu \mathrm{m}$ pore membrane, washed three times by centrifugation, and freeze-dried. Microspheres were stored at $4{ }^{\circ} \mathrm{C}$ until needed.

Particle size and morphology were analyzed by scanning electron microscopy (SEM Phillips 505, Amsterdam, Holland). Samples were gold-sputtered with an Edwards Sputter Coater S150B (Crawley, England). Drug loading was determined by dissolution of PLGA microspheres followed by HPLC/UV/FLUO. Briefly, $10 \mathrm{mg}$ of loaded microspheres was added to $10 \mathrm{~mL}$ of dimethylsulfoxide and stirred until completely dissolved. The chromatographic system consisted of a Waters 2695 Separations Module, Waters 996 DAD detector set at $228 \mathrm{~nm}$, and Waters 2475 Multiwavelength Fluorescence detector (Milford, Massachusetts, USA) set at excitation and emission wavelengths of 280 and $325 \mathrm{~nm}$, respectively. The chromatographic column was a C18 Synergi 4- $\mu$ m Hydro-RP Phenomenex column, $150 \times 4.6 \mathrm{~mm}$ (Torrance, California, USA), and the mobile phase was methanol-0.25 M ammonium acetate (60:40) at a flow rate of $0.8 \mathrm{~mL} / \mathrm{min}$. Drug loading was expressed as mg LA/100 mg microspheres.

\section{Stability Test of Leuprolide Acetate Solutions}

LA was dissolved in PBS or acetate buffer at two concentration levels, a low concentration level $(0.01-0.02 \mathrm{mg} / \mathrm{mL}$ ) simulating the initial concentration (time zero) of LA in the external medium of the in vitro release assay, and a high concentration level $(0.1-0.2 \mathrm{mg} / \mathrm{mL})$ mimicking the final cumulative amount of released LA. PLGA was added to some of the specimens at a constant PLGA-LA ratio, similar to that found in the microspheres. The flasks were appropriately sealed to avoid evaporation and incubated in an oven at $37 \pm 0.5^{\circ} \mathrm{C}$ for 31 days. The $\mathrm{pH}$ was measured, and aliquots were withdrawn at fixed times and analyzed by HPLC. Samples were vortexed for $1 \mathrm{~min}$ at the beginning of the test and before each aliquot withdrawal.

\section{In Vitro Release Test with Vertical Diffusion Cells}

A Franz cell system (Hanson model 57-6M Manual StartUp diffusion cell test system, Chatsworth, California, USA) was built as follows. A dialysis membrane (molecular cutoff 12,400 Da, Arthur Thomas Co., Philadelphia, Pennsylvania, USA) was placed on the upper donor chamber of the diffusion cell, separating this compartment from the receptor chamber. An accurately weighed quantity of LA-loaded microspheres $(10 \mathrm{mg})$ was placed on the membrane using a slab with an area of $1.77 \mathrm{~cm}^{2}$ and thickness of $1.2 \mathrm{~mm}$. One milliliter of $\mathrm{pH} 7.4150 \mathrm{mM}$ PBS buffer containing $0.1 \% \mathrm{w} / \mathrm{v}$ sodium azide and $0.05 \%$ w/v Tween 80 (PBS-T buffer) to prevent microsphere contamination and agglomeration, respectively, was added on the membrane. In another set of experiments, acetate buffer ( $\mathrm{pH} 4.0$ ) with $0.05 \% \mathrm{w} / \mathrm{v}$ Tween 80 was used (acetate-T buffer). The receptor chamber was completely filled with the corresponding buffer, wetting the membrane and the microspheres. The acrylic top plate was tightly sealed to avoid evaporation. At fixed intervals, 
Table 1. pH Variation During LA Incubation at $37^{\circ} \mathrm{C}$ in pH 4.0 Acetate and pH 7.4 Phosphate Buffers with and without PLGA at Two Concentrations

\begin{tabular}{lccccc}
\hline & & \multicolumn{4}{c}{ pH } \\
\cline { 2 - 6 } Buffer & Sample & Day 7 & Day 14 & Day 21 & Day 31 \\
\hline \multirow{4}{*}{$\begin{array}{l}\text { PBS } \\
\text { Phospate } \\
\text { Buffer }\end{array}$} & LA low & 7.3 & 7.3 & 7.3 & 7.3 \\
\cline { 2 - 6 } & LA high & 7.3 & 7.2 & 7.3 & 7.3 \\
\cline { 2 - 6 } & PLGA & 6.8 & 6.5 & 6.2 & 6.0 \\
\cline { 2 - 6 } & LA+PLGA low & 7.3 & 7.2 & 7.2 & 7.2 \\
\cline { 2 - 6 } & LA+PLGA high & 6.8 & 6.5 & 6.3 & 6.1 \\
\hline \multirow{4}{*}{$\begin{array}{l}\text { AB Acetate } \\
\text { Buffer }\end{array}$} & LA low & 4.2 & 4.2 & 4.2 & 4.2 \\
\cline { 2 - 6 } & LA high & 4.2 & 4.2 & 4.2 & 4.2 \\
\cline { 2 - 6 } & PLGA & 4.2 & 4.2 & 4.1 & 4.0 \\
\cline { 2 - 6 } & LA+PLGA low & 4.2 & 4.2 & 4.2 & 4.2 \\
\cline { 2 - 6 } & LA+PLGA high & 4.2 & 4.2 & 4.1 & 4.0 \\
\hline
\end{tabular}

aliquots were withdrawn from some cells and replenished with freshly prepared buffer. In some series of experiments with PBS, the volume of the external medium was completely removed and replenished with freshly prepared buffer. Tests were performed in triplicate at $37 \pm 0.5^{\circ} \mathrm{C}$ and $500 \mathrm{rpm}$. Samples were analyzed using the previously described HPLC/UV/FLUO conditions. The cumulative percentage released was calculated, and the mean values and standard deviations were reported.

\section{In Vitro Release Test with Rotating Bottle Apparatus}

A rotating bottle apparatus (Alycar, Buenos Aires, Argentina) that met NF XIV specifications was adapted for slow rate control. Bottles were clipped into a rotating shaft moving at $3 \mathrm{rpm}$ that was immersed in a water bath at $37 \pm 0.5^{\circ} \mathrm{C}$. Ten milligrams of accurately weighed LA loaded microspheres was filled into a dialysis tube (flat width: $2.54 \mathrm{~cm}$, length: $10 \mathrm{~cm}$, molecular cutoff: $12,400 \mathrm{Da}$ ) embedded in $3.0 \mathrm{~mL}$ PBS-T buffer. Both ends of the dialysis tubes were fastened with plastic seals. The dialysis bags were placed inside glass test tubes containing $77.0 \mathrm{~mL}$ of PBS-T buffer. The neck of each tube was sealed with silicone to prevent any liquid exchange with the external medium. Two-milliliter samples were withdrawn at specified intervals from some tubes, and from others, the complete volume was removed. In both cases, freshly prepared buffer was used to replenish. Tests were performed in triplicate, and samples were analyzed by HPLC/UV/FLUO.

\section{RESULTS AND DISCUSSION}

\section{Stability of Leuprolide Acetate in Different Media}

To study LA stability in different release media as a function of time, the drug was tested at two concentration levels in two buffer solutions, PBS and acetate buffer. In

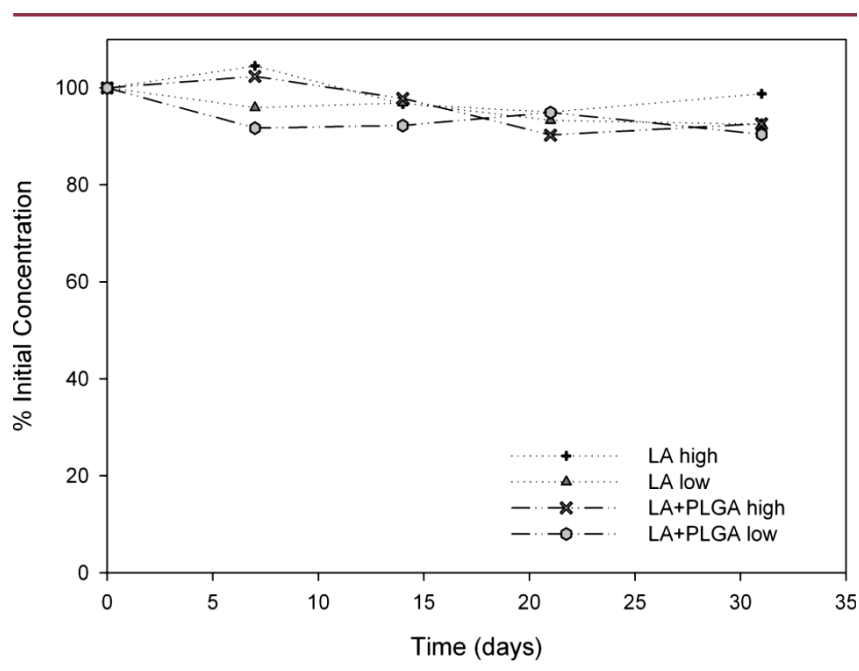

Figure 1. Stability of LA during incubation at $37^{\circ} \mathrm{C}$ in pH 7.4 PBS. Results are expressed as percentage of initial concentration $(n=3)$.

this way, the entire range of LA concentrations expected in an in vitro release assay was considered. The $\mathrm{pH}$ was also monitored in the bulk medium to relate $\mathrm{pH}$ changes to LA stability. Although the change in $\mathrm{pH}$ was controlled by buffer capacity (Table 1), the stability of the drug depended on the release medium. In PBS, LA was stable for at least 30 days at the highest concentration studied (Figure 1). A 10\% LA loss was observed throughout time upon dilution or PLGA addition. As shown in Table 1, only PLGA addition at the highest level produced a $\mathrm{pH}$ decrease of 1.4 units. On the other hand, no $\mathrm{pH}$ variations were observed in any of the samples under acidic conditions. However, LA proved to be unstable in acetate buffer, especially in the presence of PLGA and at low concentrations (Figure 2). $\mathrm{LA}$ incubated at acidic $\mathrm{pH}$ may suffer different degradation pathways according to temperature and relative LA concentration, as already reported (23). Yet, LA release

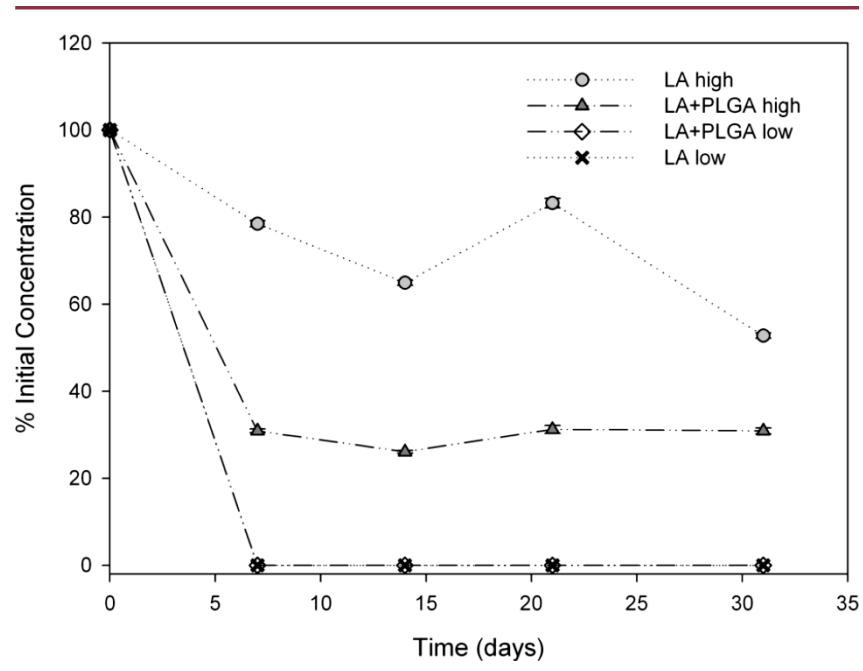

Figure 2. Stability of LA during incubation at $37^{\circ} \mathrm{C}$ in pH 4.0 acetate buffer. Results are expressed as percentage of initial concentration $(n=3)$. 


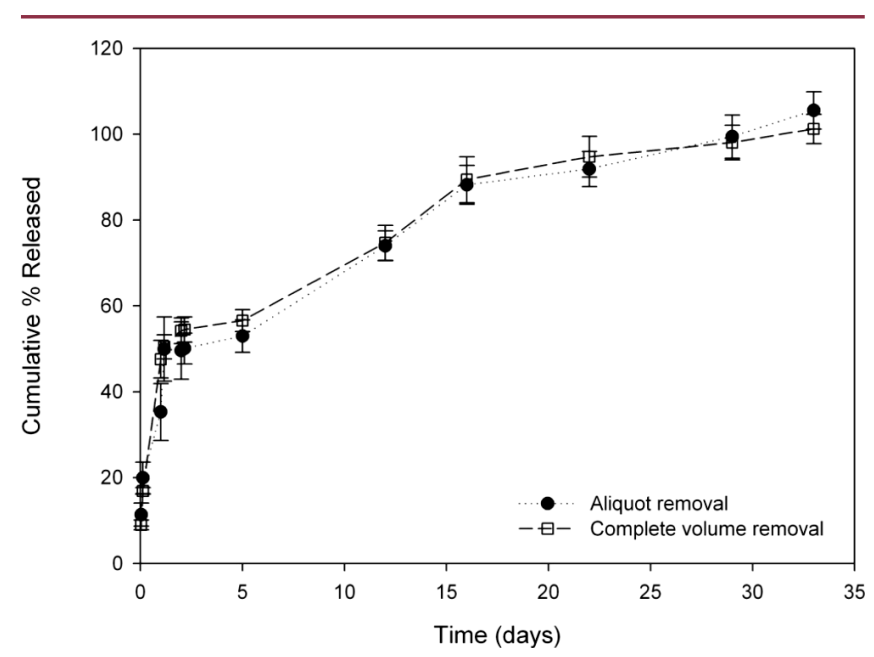

Figure 3. Cumulative in vitro release of leuprolide from PLGA microspheres using Franz diffusion cells and $\mathrm{pH} 7.4 \mathrm{PBS}$ at $37^{\circ} \mathrm{C} ;(\bullet)$ aliquots removed at fixed intervals, (ם) the complete volume of the external medium removed $(n=3)$.

assays performed in $\mathrm{pH} 4.0$ acetate buffer have been reported (24), probably in an attempt to mimic the acidic environment inside PLGA microspheres, as will be further discussed.

\section{In Vitro Release Testing: Vertical Diffusion Cells and Rotating Bottle Apparatus}

Release profiles obtained with vertical diffusion cells were clearly different depending on the release medium, phosphate or acetate, but no differences were found whether all of the external medium was replaced or an aliquot was withdrawn (Figure 3), which confirms that sink conditions had been maintained. The cumulative percentage released in PBS buffer was $105.5 \pm 4.3 \%$ for aliquot removal and $101.2 \pm 3.4 \%$ for complete volume replacement. A triphasic release profile was observed upon incubation in PBS buffer with $50 \%$ of the drug released in the first $24 \mathrm{~h}$. This kind of triphasic profile has been described previously for PLGA microspheres using different devices $(3,17,18)$. In this case, it is hard to assume that the high burst observed is only due to superficial, nonencapsulated LA release. PLGAs of different molecular weights are naturally glassy; their $T_{\mathrm{g}}$ values vary according to their physicochemical structures above physiological temperature. In a rubbery state, the mobility of polymer chains and drug molecules increases, resulting in higher drug release rates. An LA cumulative release of $86 \%$ after $30 \mathrm{~h}$ at $50^{\circ} \mathrm{C}$ from microspheres made of $8,600 \mathrm{MW}$ PLGA (50:50) with a $T_{g}$ of $40.04{ }^{\circ} \mathrm{C}$ has been reported (10). Even though our tests were performed at $37^{\circ} \mathrm{C}$, we speculate that a glassy-rubbery transition of the low molecular weight fraction of the polymer (7,000-17,000 Da) might have enhanced drug diffusion through the PLGA matrix increasing the burst effect. The short lag phase commonly assigned to erosion of the polymeric matrix can be

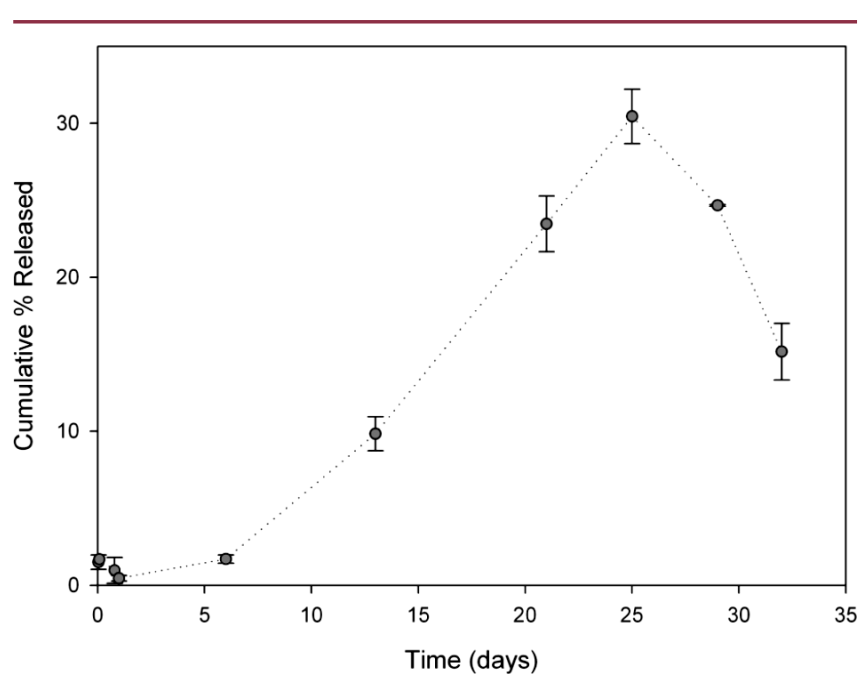

Figure 4. Cumulative in vitro release of leuprolide from PLGA microspheres using Franz diffusion cells and pH 4.0 acetate buffer at $37^{\circ} \mathrm{C}(n=2)$.

explained by the low molecular weight of the PLGA used to prepare the microspheres together with the high water solubility of the encapsulated peptide. In general, PLGA release devices are considered bulk-erosion delivery forms (25). However, it is assumed that in microspheres made of a low molecular weight polymer, the phenomenon that rules drug release is diffusion. Surface pores and cavities filled with medium in the microsphere matrix are pathways for molecules, both monomer and drug. In our case, diffusion was the key point that defined release rates and profiles. The use of a system with a low molecular weight polymer and the developed concentration gradient aided rapid drug diffusion. The high final cumulative percentage confirms drug stability throughout the assay. This can be explained by a fluid access of buffer to the easily eroded polymeric system, which prevents the formation of a low $\mathrm{pH}$ environment that would affect drug stability as described above.

On the other hand, a maximum of $30.4 \pm 1.8 \%$ was released after 25 days in acetate buffer, afterward decreasing to a cumulative $15.2 \pm 1.8 \%$ by the end of the assay (Figure 4). In this case, no burst release was obtained; instead, a lag time was observed in the first week, followed by a first-order release and a post-maximum decrease. The generation of an acidic environment inside PLGA microspheres has been studied extensively in vitro with $\mathrm{pH}$ sensitive probes $(26,27)$. The degradation of the polymer promotes $\mathrm{pH}$ decrease, which varies from very low (approximately $\mathrm{pH} 1.5$ ) at the center of the microsphere to higher values at its boundaries. Our results show that LA is unstable in acetate buffer at low concentrations and especially in the presence of PLGA (Table 1), which is in accordance with the corresponding release profile (Figure 4). In fact, although buffer seems to regulate the medium $\mathrm{pH}, \mathrm{LA}$ degradation at $\mathrm{pH} 4$ is most probably the cause of the low final cumulative percentage released and 


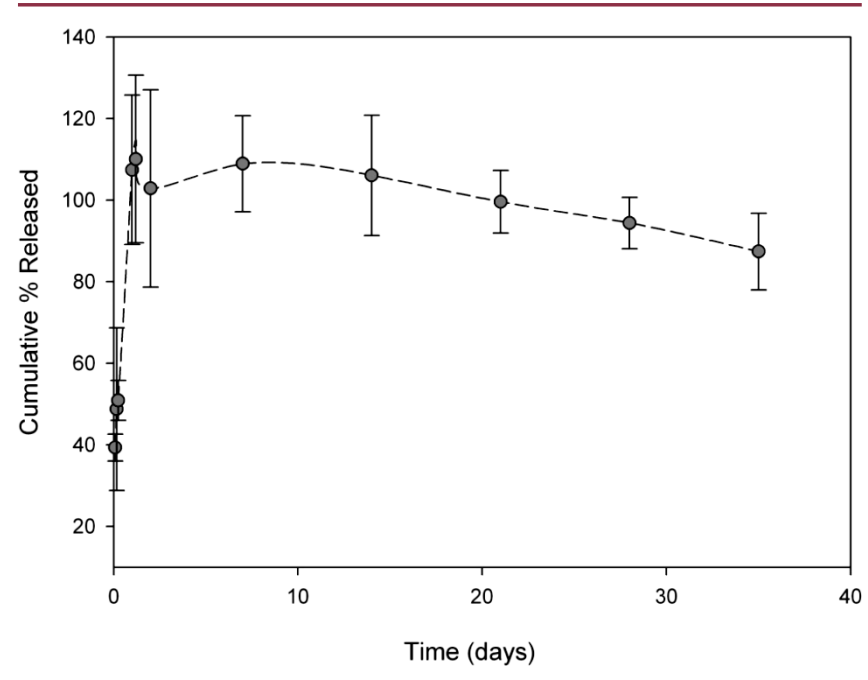

Figure 5. Cumulative in vitro release of leuprolide from PLGA microspheres using rotating bottle apparatus and $\mathrm{pH} 7.4 \mathrm{PBS}$ at $37^{\circ} \mathrm{C}$. Aliquots removed at fixed intervals $(n=2)$.

its subsequent decrease. As previously mentioned, no LA degradation was found in $\mathrm{pH}$ 7.4 PBS buffer, either in the stability test or in the in vitro release test, which led us to choose this neutral isotonic buffer for further assays.

Release profiles obtained with the rotating bottle apparatus were markedly different from those obtained with VDC. Though the cumulative percentage released was $110.1 \pm 20.5 \%$, the pattern did not fit a triphasic model (Figure 5). A high percentage of the drug was rapidly released in the burst phase, and complete release was achieved early during the test. High variability among individual values was also observed throughout the assay. Similar results were obtained either by removing aliquots or by complete volume replacement (data not shown). The high surface area available for multidirectional diffusion and the slow but constant movement may have promoted an accelerated drug migration, while the high dilution increased the variability of data.

Although erosion and swelling may occur according to the characteristics of each polymer, diffusion is always involved in the release mechanism. The rotating bottle apparatus promotes diffusive release, and the effect of LA high water solubility can be magnified in this device. In fact, microspheres move freely inside the dialysis sac at a very low speed, which prevents aggregation. In VDC, though microspheres do not move, they are placed in the buffered donor compartment in contact with a smooth surface, contrary to magnetically agitated dialysis bags (5). In this case, the release is actually a two-step process, the diffusion of LA through the polymer matrix and its subsequent diffusion from donor to receptor chamber. Therefore, diffusion cells may resemble in vivo behavior more closely, as microspheres are confined to an area but fully imbibed in release medium. When tested for a mucosal delivery system, this device simulated well the quantities of water on mucosal surfaces in vivo (9). As described by Klose et al. (28), when the drug is released from the microsphere, it encounters tissue instead of a medium without hindrance. To mimic what happens in vivo, there should be a balance between totally dispersed particles and lump-like aggregation. In our opinion, Franz diffusion cells seem to fulfill this requirement.

\section{CONCLUSIONS}

Franz diffusion cells and a rotating bottle apparatus were tested in an attempt to find an in vitro release method that may mimic the in vivo behavior of biopolymer-based delivery systems for a water-soluble drug. Leuprolide acetate, which is stable at physiological conditions even in the presence of PLGA, displayed triphasic and biphasic release profiles in Franz diffusion cells and the rotating bottle apparatus, respectively.VDC, where the microspheres are partially confined but sink conditions are still maintained, appeared to be a suitable alternative to the existing USP Apparatus 4. Further investigations will continue with different biodegradable polymers tested under similar conditions and contrasted to in vivo profiles to establish if Franz diffusion cells can be considered as an alternative in vitro method for predicting in vivo behavior of prolonged-release biodegradable systems.

\section{ACKNOWLEDGMENTS}

The authors would like to thank Dr. Nora François and Dr. Marta Daraio for technical assistance in the calculations. This work was supported by PTA grant 125/08 from the National Institute of Industrial Technology, Argentina.

\section{REFERENCES}

1. Martinez, M.; Rathbone, M.; Burgess, D.; Huynh, M. In vitro and in vivo considerations associated with parenteral sustained release products: A review based upon information presented and points expressed at the 2007 Controlled Release Society Annual Meeting. J. Control. Release 2008, 129 (2), 79-87.

2. Rathbone, M. J.; Martinez, M. N.; Huynh, M.; Burgess, D. J. CPS/AAPS Joint Workshop on Critical Variables in the In Vitro and In Vivo Performance of Parenteral Sustained-Release Products. Dissolution Technol. 2009, $16(2), 55-56$.

3. Zolnik, B. S.; Leary, P.E.; Burgess, D. J. Elevated temperature accelerated release testing of PLGA microspheres. J. Control. Release 2006, 112 (3), 293-300.

4. The United States Pharmacopeia and National Formulary USP 32-NF 27; The United States Pharmacopeial Convention, Inc.: Rockville, MD, 2009.

5. D'Souza, S.; De Luca, P. Development of a dialysis in vitro release method for biodegradable microspheres. AAPS PharmSciTech 2005, 6 (2), E323-E328.

6. D'Souza, S.; De Luca, P. Methods to assess in vitro drug release from injectable polymeric particulate systems. Pharm. Res. 2006, 23 (3), 460-474. 
7. Franz, T.J. Percutaneous absorption. On the relevance of in vitro data. J. Invest. Dermatol. 1975, 64, 190-195.

8. Bertram, U.; Bodmeier, R. In situ gelling, bioadhesive nasal inserts for extended drug delivery: In vitro characterization of a new nasal dosage form. Eur.J. Pharm. Sci. 2006, 27 (1), 62-71.

9. Moebus, K.; Siepmann, J.; Bodmeier, R. Alginatepoloxamer microparticles for controlled drug delivery to mucosal tissue. Eur. J. Pharm. Biopharm. 2009, 72 (1), 42-53.

10. Shameem, M.; Lee, H.; DeLuca, P. A short-term (accelerated release) approach to evaluate peptide release from PLGA depot formulations. AAPS PharmSci. 1999, 1 (3), 1-6.

11. Du, L.; Cheng, J.; Chi, Q.; Qie, J.; Liu, Y.; Mei, X. Biodegradable PLGA microspheres as a sustained release system for a new luteinizing hormone-releasing hormone (LHRH) antagonist. Chem. Pharm. Bull. 2006, 54 (9), 1259-1265.

12. Ogawa, Y.; Yamamoto, M.; Okada, H.; Yashiki, T.; Shimamoto, T. A new technique to efficiently entrap leuprolide acetate into microcapsules of polylactic acid or copoly(lactic/glycolic) acid. Chem. Pharm. Bull. 1988, 36 (3), 1095-1103.

13. Okada, H. One- and three-month release injectable microspheres of the $\mathrm{LH}-\mathrm{RH}$ superagonist leuprorelin acetate. Adv. Drug Deliv. Rev. 1997, 28 (1), 43-70.

14. Luan, X.; Bodmeier, R. Modification of the tri-phasic drug release pattern of leuprolide acetate-loaded poly(lactide-co-glycolide) microparticles. Eur. J. Pharm. Biopharm. 2006, 63 (2), 205-214.

15. Luan, X.; Skupin, M.; Siepmann, J.; Bodmeier, R. Key parameters affecting the initial release (burst) and encapsulation efficiency of peptide-containing poly(lactide-co-glycolide) microparticles. Int. J.Pharm. 2006, 324 (2), 168-175.

16. Faisant, N.; Akiki, J.; Siepmann, F.; Benoit, J. P.; Siepmann, J. Effects of the type of release medium on drug release from PLGA-based microparticles: Experiment and theory. Int. J. Pharm. 2006, 314 (2), 189-197.
17. Zolnik, B. S.; Burgess, D. J. Evaluation of in vivo-in vitro release of dexamethasone from PLGA microspheres. J. Control. Release 2008, 127 (2), 137-145.

18. Kumar, R.; Palmieri, M. J. Points to consider when establishing drug specifications for parenteral microspheres. AAPS J. 2010, 12 (1), 27-32.

19. Zolnik, B. S.; Burgess, D. J. Effect of acidic pH on PLGA microsphere degradation and release. J. Control. Release 2007, 122 (3), 338-344.

20. Anderson, J.M. Biological Responses to Materials. Annu. Rev. Mater. Res. 2001, 31 (1), 81-110.

21. Zolnik, B.S.; Raton, J. L.; Burgess, D. J. Application of USP Apparatus 4 and In Situ Fiber Optic Analysis to Microsphere Release Testing. Dissolution Technol. 2005, 12 (2), 11-14.

22. Voisine, J. M.; Zolnik, B. S.; Burgess, D. J. In situ fiber optic method for long-term in vitro release testing of microspheres. Int. J. Pharm. 2008, 356 (1-2), 206-211.

23. Stevenson, C. L.; Leonard, J. J.; Hall, S. C. Effect of peptide concentration and temperature on leuprolide stability in dimethyl sulfoxide. Int.J. Pharm. 1999, 191 (2), 115-129.

24. D’Souza, S. S.; Faraj, J. A.; De Luca, P. A model-dependent approach to correlate accelerated with real-time release from biodegradable microspheres. AAPS PharmSciTech 2005, 6 (4), E553-E564.

25. Faisant, N.; Siepmann, J.; Benoit, J. P. PLGA-based microparticles: elucidation of mechanisms and a new, simple mathematical model quantifying drug release. Eur. J. Pharm. Sci. 2002, 15 (4), 355-366.

26. Fu, K.; Pack, D. W.; Klibanov, A. M.; Langer, R. Visual evidence of acidic environment within degrading poly(lactic-co-glycolic acid) (PLGA) microspheres. Pharm. Res. 2000, 17 (1), 100-106.

27. Li, L.; Schwendeman, S. P. Mapping neutral microclimate $\mathrm{pH}$ in PLGA microspheres. J. Control. Release 2005, 101 (1-3), 163-173.

28. Klose, D.; Azaroual, N.; Siepmann, F.; Vermeersch, G.; Siepmann, J. Towards More Realistic In Vitro Release Measurement Techniques for Biodegradable Microparticles. Pharm. Res. 2009, 26 (3), 691-699. 